\title{
Discussion on Virtual Experiment in Applied Undergraduate Numerical Control Teaching Dongfang $\mathrm{Hu}$, Chang Liu and Yichen $\mathrm{Li}$
}

(School of Mechanical and Electrical Engineering, Department of Mechanical Engineering, Henan University of Science and Technology, Henan, Luoyang, 471000)

\begin{abstract}
According to the training target and present situation of applied undergraduate talents in higher education, this paper analyzes the general problems of the traditional teaching model of engineering in colleges and universities at present stage and puts forward the view that virtual experiment should apply to the applied undergraduate numerical control teaching. Based on the characteristics of virtual experiment teaching mode, the composition of virtual simulation experiment teaching system is introduced. Then collecting the data of college students through the questionnaire and after that the results of the questionnaire are analyzed and summarized, which provide the theoretical basis for the reform of the teaching methods of cultivating applied talents.
\end{abstract}

Keywords: Application-Oriented bachelor degree; Virtual experiment teaching; Virtual simulation; CNC teaching; Engineering teaching

\section{虚拟实验在应用型本科数控教学中的探讨}

\author{
胡东方，刘畅，李奕辰 \\ (河南科技大学 机电工程学院、机械工程系, 河南 洛阳 471000)
}

摘要: 针对高等教育应用型本科人才培养目标及现状, 分析了现阶段高校工科传统教学模式的普遍问题, 提出了虚拟实 验应用于应用型本科数控教学中的观点。基于虚拟实验教学模式的特点, 介绍了虚拟仿真实验教学系统的组成。通过调查问 卷的形式, 对在校大学生进行数据采集, 并对调查问卷的结果进行了分析与总结, 为努力做好培养应用型人才的教学方式的 改革提供了理论依据。

关键词: 应用型本科; 虚拟实验教学; 虚拟仿真; 数控教学; 工科教学

引言

随着 “中国制造 2025” 的全面部署, 我国由制造大国向制造强国的转变已经成为新时期的发展需要 [1]。数控机床所提供的制造装备及其控制的智能化在这一时代背景下显的尤为关键。数控水平的高低已 经成为衡量一个国家制造业水平的重要标志 [2-3]。随着我国制造业的转变, 企业对于人才的要求也随着 科学技术的进步而日益严格, 懂理论、懂技能、懂创新的 “三懂人才” 成为企业发展的立足之根。为应对 这一情况, 目前国内部分高校已积极作出调整, 利用翻转课堂、慕课 [4]等方式进行对现有课程资源的补 充, 但由于教学资源等因素的限制, 很多高校在学生的实践教学环节中仍处于原地踏步阶段 [5], 纸上得 来终觉浅, 绝知此事要躬行, 缺少实践的教学模式将难以培养出高技术数控人才。本文基于此背景下, 将 虚拟实验应用到高校的教学中, 通过比较虚拟实验与传统实验的教学效果展开研究 [6]。

\section{1 传统教学现状及问题}

\section{1 传统数控教学的一般模式}

在数控教学体系中, 教师将知识传给学生的同时, 学生也将对知识的理解反馈给教师。目前国内工科 院校虽然存在教学资源与教学手段的差异, 但教学模式却极其相似, 如图 1 所示 


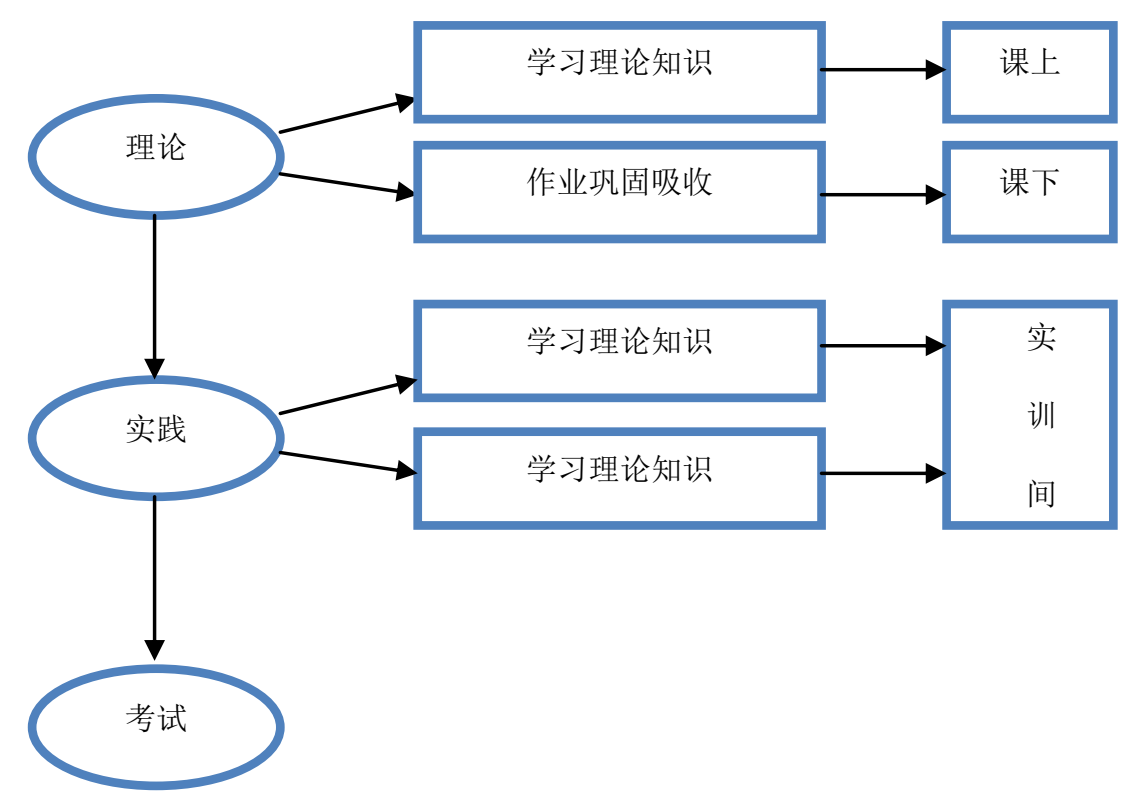

图 1 传统数控教学模式

(1) 理论课程的学习, 包括教师教授课本上的理论知识和通过课下布置作业的方式令学生对所学知识 予以吸收;

(2) 数控教学的实践, 主要包括在实训车间内实习教师对学生进行认知实习方面知识的普及和给予学 生实际操作的指导, 旨在培养学生的动手能力, 强化对理论知识的理解。

(3) 考试, 根据整门课程的教学计划编写数控技术这门课程的理论试卷和制定实机操作考试, 主要考 察学生的理论学习和实际动手能力。

\section{2 数控教学中存在的问题}

\subsection{1 授课方式较为单一}

数控技术是一门综合性学科, 具有非常强的系统性、连贯性。当代大学生求知欲强, 学习积极性高, 但也存在浮躁心理, 如果不能激发其学习兴趣, 就会对数控技术这门课程产生抵触情绪; 其次, 现代数控 技术高速发展, 书本知识无法跟上现代科技的发展脚步, 对于工科而言这一点非常致命, 唯有放眼于全球 的高端科技，构思与时俱进的先进制造理念，才能给我国的制造业注入新鲜的血液，推动科技发展; 另一 方面, 当授课教师的教学手段单一无法调动课堂的积极性时, 无疑是给本来就难懂的课程更加雪上加霜, 导致学生的理论知识储备不足, 故将难以培养出优秀的数控人才。

\subsection{2 授课内容无法满足实际需要}

随着应用型本科这一概念的提出, 其主要的教学要求包括专业知识的学习和专业能力的培养, 专业能 力与就业能力和创新能力有关, 它是应用型本科的核心目标。就目前的现状来看, 很多高校在教学模式中 还没有形成这一理念, 其授课形式仍旧是以书本为导向的照本宣科式教学方法, 这样便会导致课上的知识 内容无法匹配学生在今后工作中的实际需要。

\section{2 .3 教学资源的不足}

高校实践教学是培养学生创新能力的重要环节, 学生创新能力的培养已经被提升到国家战略的高度, 但目前实验设备的严重滞后是国内高校的普遍弊端, 实践项目无法同步反应当前的前沿科技, 令实践教学 的发展举步维艰 [7]。在教学时间一定的情况下, 每名学生能够亲自上机操作的时间少之又少, 多半是停 
留在用眼睛看, 而不是用手做的冦尤境地。众所周知, 建设一条完整的实习车间花费极高, 性能优越的机 床动辄几十万，普通院校几乎无法承担如此大的经费投入，若不及时对数控教学进行改革，更新原有的教 学模式, 将严重影响教学质量和人才的培养。

\section{2 虚拟实验对于数控教学的重要性}

虚拟实验是指借助于多媒体仿真和虚拟现实等技术在计算机上所进行的对传统实验各操作环节的模 拟和仿真 [8]。由于在实际教学环境中, 大多数高校都缺少实验设备与场地, 致使学生难以亲自动手实践, 这些因素制约着学生能力的发展, 所以将虚拟实验引进课堂教学是极其重要的 [9]。虚拟实验依托虚拟仿 真技术利用电脑软件与硬件相结合，在计算机上进行模拟，仿真各种实验的技术，与传统教学模式相比虚 拟实验具有显著地优势, 其主要优势表现在如图 2 所示

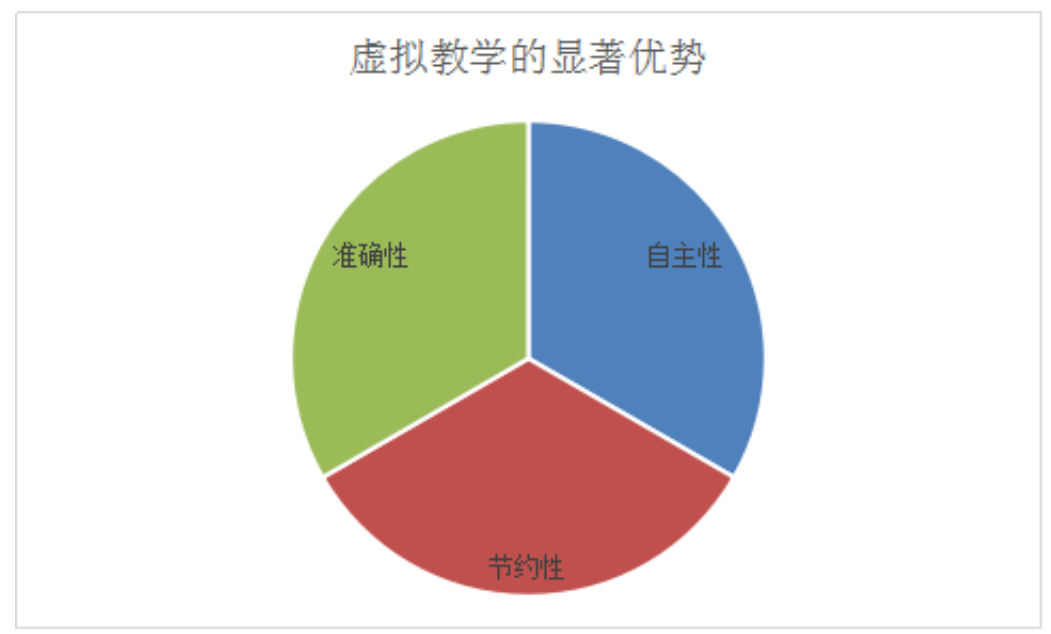

图 2 虚拟教学的显著优势

（1）在虚拟实验中，学生可以拥有比传统实验更加广阔的学习自主权，同时新型授课方式会极大激发 学生的学习兴趣, 借助虚拟实验室, 学生可以构思与模拟自己感兴趣的实践课题, 通过开放式的教学资源, 自由构建实验环境和实验数据, 这显然是传统教学模式所不具备的;

（2）教学成本的降低，可以为高校减少购置传统教学所需的数控机床等实践设备的费用，依托虚拟实 验室就可以达到实践需求, 在降低设备的维修和保养成本时也能避免传统实践大型装备对学生可能带来的 安全隐患;

（3）学生可不受实验环境等客观因素的影响进行实验, 只需通过提供实验数据等参数便可以让计算机 模拟结果，在节省实验材料的同时也能避免传统设备因机械部件达不到标准需要而导致的误差。

总的来说, 虚拟仿真实验利用计算机达到教学要求, 通过实现虚拟制造和虚拟实验分析, 大大节省了 人力、财力和时间。学生可以在虚拟环境中反复学习充分发挥创新设计思维, 通过数字化技术的应用, 拉 近数控教学内容与实际企业需要的距离。

\section{3 虚拟实验教学系统的组成}

数控教学的虚拟实验主要包括数控机床的基本操作、插补原理的实验、数控加工的实验等。通过开放 式的互联网平台, 可以下载数控加工设备的相关参数后运用几何建模方法采用事物虚化的手段建立虚拟化 的机械产品模型, 因其这些虚拟模型在构建后已经具有跟现实机床同等的物理属性, 所以在虚拟实验的时 
候足以满足教学需要, 该系统一般有以下几部分组成 [10]。

\section{1 用户设计界面}

在虚拟实验系统中，该界面的提供主要是为了方便使用者对于实验环境使用的初次准备，如机床的类 别的选用、工件材料, 材质、刀具的参数选用等。通过计算机模拟出的虚拟场景, 不仅能让使用者最大程 度上还原真实条件下的试验情景还可以规范使用者的操作规范

\section{2 实验仿真界面}

数控加工仿真利用计算机模拟实际的加工过程, 是检验数控加工程序是否合理的有力保障。建立虚拟 仿真系统通常采用 WINDOWS XP 系统, 仿真工具一般选用 UG 等 CAD/CAM 三维开发软件。使用者通过在 UG 上绘制零件模型, 针对加工对象的材料、性能要求等方面, 选择合适的加工方法和加工参数, 并借以 UG/CAM 模块生成的 CLSF 文件及 NC 代码, 通过写入 NC 代码可在 VERICUT 或斯沃仿真软件中实现刀具的切削仿真。

\section{3 实验结果反馈界面}

使用者在实验虚拟实验系统的时候，计算机会自动保存使用者的实验类型并予以实时记录，同时还会 保存使用者在进行仿真实验时所使用到的实验参数, 包括: 刀具的选用, 插补的计算等并记录其操作流程 为二次实验提供理论依据。在实验完成后，系统会自动生成可供打印的实验报告，使用者通过阅读实验报 告与实验预期形成闭环反馈，总结实验经验、分析实验中出现的问题，这对使用者的实验能力提升有着极 大的帮助。

\section{4基于调查问卷的比较}

\section{1 问卷设计}

此次问卷调查采用自编问卷的形式进行调查, 调查的问题包括学生对虚拟实验应用于数控教学中的认 识, 以及虚拟实验对于他们的学习帮助程度等方面。问题类型包括两种：单选题和问答题。

\section{2 结果分析}

针对虚拟实验对数控教学的可行性进行调查, 调查对象为我校 2014-2015 级机械制造及其自动化专业 共计 100 名学生, 调查方式为随机抽样。共发放调查问卷 100 份, 回收问卷 95 份, 其中无效问卷 3 份。 具体的问卷有效率如表 1 所示。

表 1 调查问卷的回收情况

\begin{tabular}{cccccc}
\hline 年级 & 发放总数 & 回收数目 & 有效数目 & 回收率 & 有效率 \\
\hline 2014 & 50 & 48 & 47 & $96 \%$ & $98 \%$ \\
2015 & 50 & 47 & 45 & $94 \%$ & $96 \%$ \\
\hline
\end{tabular}

\section{3 问卷内容}

问卷内容及调查结果如表 2 所示

表 2 基于虚拟实验教学应用的问卷调查

\begin{tabular}{cccc}
\hline 调查问题 & A 选项比率 & B 选项比例 & C 选项比例 \\
\hline 1. 引进虚拟实验的态度 & 感兴趣 $85 \%$ & 兴趣一般 $10 \%$ & 不感兴趣 $5 \%$
\end{tabular}




\begin{tabular}{|c|c|c|c|}
\hline \multirow{2}{*}{ 2.虚拟实验的优势 } & 实验设备与时俱进 & 节省资金 $10 \%$ & 科技感 $60 \%$ \\
\hline & $30 \%$ & & \\
\hline \multirow[t]{2}{*}{ 3.虚拟实验的劣势 } & 实验效果过于理想化 & 和实际体验感觉不一样 & 虚幻 $15 \%$ \\
\hline & $15 \%$ & $70 \%$ & \\
\hline 4. 虚拟实验对学习的影响 & 很有帮助 $86 \%$ & 没有帮助 $4 \%$ & 不好说 $10 \%$ \\
\hline 5. 虚拟实验的改进方法 & 需要改进 73\% & 不需要改进 23\% & 不好说 $4 \%$ \\
\hline 6.虚拟实验能否替代传统 & 能替代 $23 \%$ & 不能替代 $67 \%$ & 不好说 $10 \%$ \\
\hline 实验 & & & \\
\hline
\end{tabular}

对该问卷调查向同学们提出的 6 个问题结果统计情况分析如下:

（1）虚拟实验的引进确实能激发学生的学习兴趣, 对虚拟实验感兴趣的学生比例多达 $85 \%$, 认为对自 己学习技能和实践能力的提高有帮助的多达 $86 \%$, 由此可知对于虚拟实验教学这一具有科技感的新鲜事物 来说, 还是很受学生们的欢迎。基于虚拟实验教学方式的引进, 高校可以打破经费和地域的发展弊端, 通 过虚拟现实技术提供给学生不限实验次数限制的实践机会，可以让学生就某一课题多次实验，从不同的实 验数据中加强对知识的吸收，提高实验效率的同时还有助于培养学生的创新能力。

(2) 在问题 5 中不难看出, $73 \%$ 的学生还是认为虚拟实验教学还是需要改进的, 因为虚拟实验教学对 大多数学生而言还是比较新鲜的, 学生单从字面意思来理解或许只能得到科技感十足这一单一的含义还远 远不够。这就需要授课教师的不断引导，打破传统教学模式 “只见课本，不见实践” 的枷锁，在完善好学 生的知识体系框架后, 应及时引领学生参与到教学实践中。任何系统平台的开发都应该走 “设计一应用一反 馈-再设计” 的这一闭环系统, 这就需要虚拟实验教学的建设要与学生紧密结合, 通过多收集学生的使用 意见或建议, 才能不断完善学生的使用需求, 促进虚拟教学实验的推广。

（3）尽管虚拟实验教学很受学生的欢迎，但通过问题 6 的调查我们发现 67\%的学生还是不愿放弃传统 实验教学, 认为虚拟实验可能在短时间内无法替代传统实验, 他们普遍认为虚拟实验模型可能在实验对象 的反应上有所缺失, 比如被实验对象本身所具备的自然属性, 摸不到的东西对他们而言可能会缺失实际技 能的积累; 同时实验结果可能过于理想化, 忽略了学生在实际操作中操作方式的不同可能给实验最终的效 果带来的差异，而这在真实的情况下是存在的，这或许是虚拟实验的局限所在。

此外，在本次调查问卷的题目中我们还设置了两道问答题，分别是（1） “虚拟实验与传统实验相结 合的教学方式是否合理”、（2）“对于虚拟实验和传统实验更倾向于哪一种”。通过对回收后问卷调查 结果的整理和分析，对于问题（1）“虚拟实验与传统实验相结合的教学方式是否合理” 这个观点的调查统 计结果如表 3 所示。5-1 分别是完全同意、较为同意、一般、较不同意、不同意五个等级。

表 3 关于虚拟实验与传统实验相结合的方式是否合理

\begin{tabular}{ccccc}
\hline 满意度 & 人数 & 百分比 & 有效百分比 & 累计百分比 \\
\hline 1 & 1 & 1.1 & 1.1 & 1.1 \\
2 & 3 & 3.3 & 3.3 & 4.4 \\
3 & 10 & 10.9 & 10.9 & 15.3
\end{tabular}


4

5
24

54

92
26.2

58.5

100
26.2

58.5

100

由表 3 可以看出, $84.7 \%$ 的受访学生比较赞同虚拟实验与传统实验相结合的教学模式, 认为能很好的 提高教学效果, 提升学生的综合素质。因此, 在实际教学中, 应使二者实践模式相结合, 取长补短从而更 好提升教学质量。

（2）关于在何种教学模式中选择虚拟实验教学, 对于这个问题将收集的整理整理后, 绘制出柱状数据, 如图 3 所示

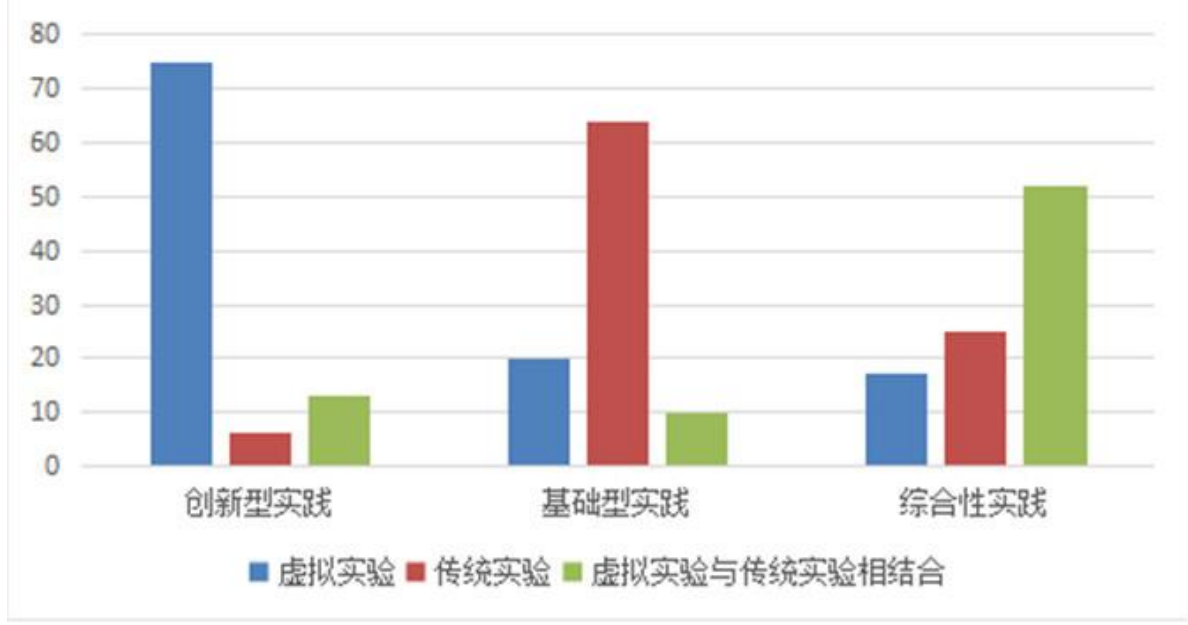

图 3 基于虚拟实验的教学要求

高校在创新型实践教学中，有 79.8\%的学生选择使用虚拟实验; 基础型实践中有 $68 \%$ 的学生选择传统 实验; 而在综合型实践中有 55\%的学生选择虚拟实验与传统实验教学相结合的方法。

综上所述，在实践教学过程中要针对实践项目的复杂程度及其各方面的条件限制从而选择最佳的教学 模式。对于简单的基础性教学为目的的实践还应以传统实践教学为主, 以此来提高学生的动手操作能力; 对于创新型实践来说, 由于实验设备本身的硬件属性可能无法满足当前教学要求, 同时现代大学生的思维 能力强, 好奇心旺盛, 此时可以借助真实环境下进行参数设定在虚拟环境下进行实践, 可以从不同的实践 思路出发探寻不同的实验结果, 此举可以在最大程度上提高学生的思想创新意识。

\section{5 结束语}

虚拟实验教学对于各高校努力探寻应用型本科教学模式的改革具有一定的指导意义，也是对传统教学 模式的一次创新升级。一方面能够有效解决高校内部实验设备不足、经费少的问题, 同时还可以满足现阶 段下培养应用型本科数控人才的时代需要。通过虚拟实验教学, 学生的学习自主性极大提高, 有利于学生 对数控技术这门课程知识的掌握, 通过实验过程可以加深对书本内所学知识的理解, 这对学生实践能力的 培养有着积极地推动作用。 


\section{Acknowledgement}

The authors gratefully acknowledge the National Superior Course and Bilingual Teaching Demonstration Course for financial support of this research work and the Project for Education and Teaching Reform of Henan University of Science and Technology (No. 2012N-007).

参考文献

[1］陈鹏，庞学光. 《中国制造 2025》与现代职业教育转型发展 [J]．教育发展研究, 2015, (17)：15-20.

[2] 蔡锐龙, 李晓栋, 钱思思. 国内外数控系统技术研究现状与发展趋势 $[J]$. 机械科学与技术, 2016, (04)： 493-500.

[3] 刘敏, 罗建. 浅析我国数控技术的现状及其发展趋势 [J]．科技创新与应用, 2016，（32）：137.

[4] 赵否，朱泓，马红亮. 高校混合教学的新机遇:MOOC 视角 [J]。教育发展研究, 2015，（17）：50-55.

[5] 刘先勇. 数控加工课程一体化教学改革的实践与评价 [A]. 中国职协 2015 年度优秀科研成果获奖论文集（上册） [C]. 2016:7.

[6] 王广官. 基于虚拟现实技术的数控车床仿真系统的研究与开发 [D]. 浙江大学, 2016.

[7] 厉成. 数控考工实习教学改革研究与实践 [J]. 教育观察 (上半月), 2016, (05)：122-123.

[8］李孝茹，刘建国. 基于虚拟的测控实验教学研究 [J]. 科教导刊(下旬)，2016，(01）：76-77.

[9] 蒙艳玫，唐治宏，董振，等. 机械工程虚拟仿真实验教学体系的研究与实践 $[\mathrm{J}]$. 实验技术与管理，2016，(05)： 109-112.

[10] 刘汉代, 赵杰, 廖志良, 等. 机械原理虚拟实验系统的设计与实现 $[J]$. 机械工程与自动化, 2016, (05)： 61-63+66.

\section{References:}

[1] Chen Peng, Pang Xueguang. Made in China 2025 and Transition Development of Modern Vocational Education [J].Exploring Education Development, 2015, (17):15-20.

[2] Cai Ruilong, Li Xiaodong, Qian Sisi. Research Present Status and Development Trend of Numerical Control System Technology at Home and Abroad [J]. Mechanical Science and Technology for Aerospace Engineering, 2016, (04):493-500.

[3] Liu Min, Luo Jian. Analysis on Present Situation and Development Trend of NC Technology in China [J].Technology Innovation and Application, 2016, (32):137.

[4] Zhao Lei, Zhu Hong, Ma Hongliang. New Opportunities of Mixing Teaching in Colleges and Universities: MOOC perspective [J].Exploring Education Development, 2015, (17):50-55.

[5] Liu Xianyong. Practice and Evaluation of Integration Teaching Reform of CNC machining curriculum [A]. 2015 Award Anthology of Outstanding Scientific Research Achievements in China Vocational Association (Volume One) [C].2016:7.

[6] Wang guangguan. Research and Development of Simulation System of CNC Lathe Based on Virtual Reality Technology [D].Zhejiang University, 2016.

[7] Li Cheng. Study of Practice of Internship Teaching Reform for NC Works [J].Survey of Education (First Half), 2016, (05):122-123.

[8] Li Xiaoru, Liu Jianguo. Experiment Teaching Study on Based on Virtual Measurement and Control [J].The Guide of Science and Education (the Last Ten-Day Period of a Month),2016,(01):76-77.

[9] Meng Yanmei, Tang Zhihong, Dong Zhen, and so on. Research and Practice on Teaching System of Virtual Simulation Experiment in Mechanical Engineering [J].Experimental 
Technology and Management, 2016, (05):109-112.

[10]Liu Daihan, Zhao Jie, Liao Zhiliang, and so on. Design and Realization of Virtual Experiment System of Mechanical Principle [J].Mechanical Engineering and Automation, 2016,(05):61-63+66. 